\title{
Solitary fibrous tumors of the prostate: A case report
}

\author{
WENYAN YANG ${ }^{1}$, FUGUANG SUN ${ }^{1}$, HONGJUN LIU ${ }^{1}$, GUANGJIAN WANG ${ }^{1}$, \\ PEIQING SHI ${ }^{1}$, ZHIQIANG SHAO ${ }^{2}$ and FENGFU GUO ${ }^{1}$ \\ ${ }^{1}$ Department of Urology, Linyi People's Hospital, Linyi, Shandong 276003; ${ }^{2}$ Department of Urology, \\ The First Affiliated Hospital of The General Hospital of the People's Liberation Army, Beijing 100037, P.R. China
}

Received August 8, 2014; Accepted April 14, 2015

DOI: $10.3892 / 01.2015 .3379$

\begin{abstract}
Increasing numbers of extrapleural solitary fibrous tumours (SFTs), including the prostate SFT, have been reported over the last 10 -years. Prostate SFT is relatively uncommon, with $<20$ cases reported in the literature worldwide. In the present study, a prostate SFT case, which was initially misdiagnosed as benign prostatic hyperplasia, is presented. The patient was subjected to three surgeries (cystoscopy and per urethra lithocystotomy, transurethral resection of the prostate and nerve-sparing retropubic radical prostatectomy) prior to SFT diagnosis. It was demonstrated that histopathological and immunohistochemical factors (positive staining for CD34 and B cell lyphoma-2 expression) were of significant diagnostic value. Thus, nerve-sparing retropubic radical prostatectomy for total resection may be the best therapeutic strategy to treat prostate SFT, allowing the preservation of sexual function and reducing the risk of locoregional recurrence.
\end{abstract}

\section{Introduction}

Solitary fibrous tumors (SFTs) were first described in 1931, as a neoplasm usually originating from the pleura (1). However, over the past 10 years, increasing numbers of extrapleural SFTs have been reported, including those of the prostate. Prostate SFT is relatively uncommon, with $<20$ cases reported in the literature worldwide $(2,3)$. According to these reports, 13 cases of prostate SFT were identified by prostate needle biopsy or transurethral resection (TUR) of the prostate. The majority of cases presented with urinary tract symptoms (4-6), and were treated by complete tumor resection [cystoprostatectomy (7), radical prostatectomy $(8,9)$, pelvic exenteration or pelvic tumor resection (10)] or enucleation and TUR (11). According to

Correspondence to: Dr Zhiqiang Shao, Department of Urology, The First Affiliated Hospital of The General Hospital of the People's Liberation Army, 51 Fucheng Road, Beijing 100037, P.R. China

E-mail: zhiqiangshao2014@163.com

Dr Fengfu Guo, Department of Urology, Linyi People's Hospital, 27 Jiefang Road, Linyi, Shandong 276003, P.R. China

E-mail: guoff9892@163.com

Key words: prostate, solitary fibrous tumour immunohistochemical analysis of the tumors, all of the cases except one were immunoreactive for CD34, and all of the cases were positive for B cell lymphoma-2 (bcl-2), CD99, $\beta$-catenin and c-kit (12-14). In addition, three SFTs demonstrated $\geq 10 \%$ p53 immunoreactivity, and three cases revealed Ki-67 rates of $\geq 20 \%$ (12).

There have also been several case reports of SFTs in the prostate. For example, a 37-year-old male presented with irritative lower urinary tract symptoms, as a result of a mass in the perineum which displaced and distorted the bulbar urethra. Following enucleation of the tumor, the patient's condition gradually returned to normal during the 2-year follow-up period. The tumor was 'patternless' with a combination of alternating hyper- and hypocellular areas, and the tumor cells were spindle-shaped with bland nuclei, having dispersed chromatin and inconspicuous nucleoli. Furthermore, the cells were markedly immunoreactive for $\mathrm{CD} 34$ and vimentin, but negative for cytokeratin AE1/AE3, smooth muscle actin, S-100 protein and desmin (15). Similarly, a 60-year-old male presenting with lower urinary tract symptoms was found to have an enlarged and hard left prostate lobe. Based on the results of histopathological and immunohistochemical analyses, including the arrangement of the tumor cells in an irregular pattern, the identification of short-spindled cells possessing meagre amounts of eosinophilic cytoplasm, the presence of bland nuclei with uniformly distributed chromatin and inconspicuous nucleoli, immunoreactivity to CD34 and bcl-2 but negative immunoreactivity to CD117, anaplastic lymphoma kinase, smooth muscle actin and progesterone receptors, a diagnosis of SFT was reached. Following treatment with nerve-sparing retropubic radical prostatectomy, the mass was well delineated with no apparent invasion of the bladder neck or pelvic wall identified (2). Finally, two males aged 66 and 69 years-old, presenting with urinary tract symptoms, were diagnosed with SFT, by transrectal needle biopsy and TUR of the prostate, in 2011. The tumors were excised with a low anterior resection. The two tumors were well-circumscribed, although a small quantity of infiltration into the prostate glands was identified. The tumors consisted of storiform bundles of bland spindle cells, which stained strongly for CD34 and vimentin, but were negative for the expression of muscle markers. Following therapy, no relapses have been reported in either of the cases, although the follow-up periods were short (16). In the present study, one significant case of SFT is reported, aimed at promoting understanding of the diagnosis and treatment of 


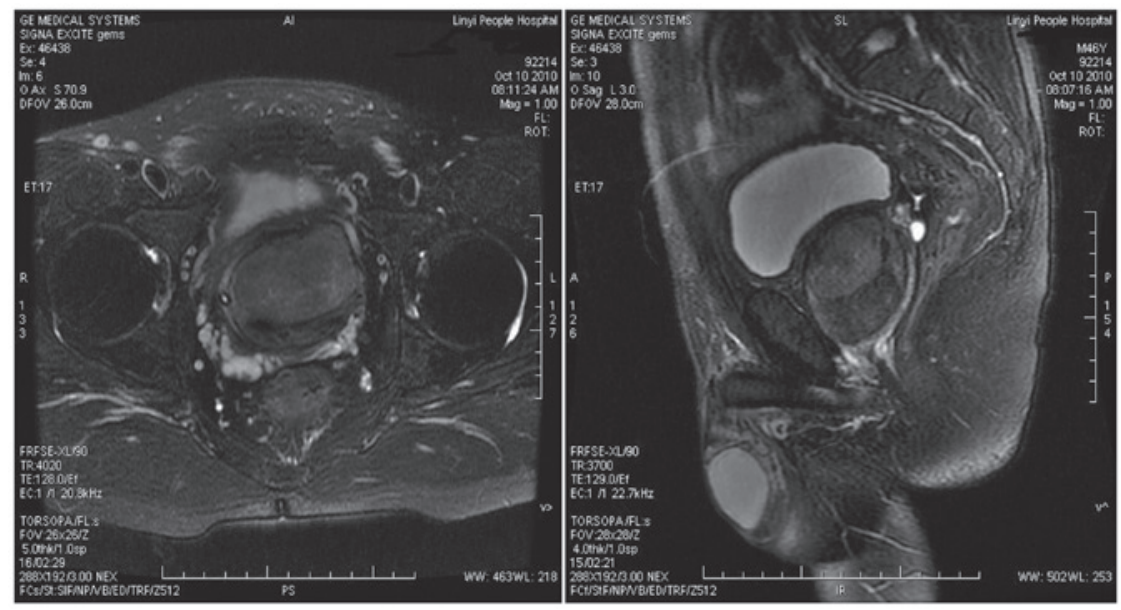

Figure 1. Magnetic resonance imaging identified a large solid mass arising from the prostate. T2WI of fat-suppression on the axial plane of abscissas (left) and anteroposterior (right).
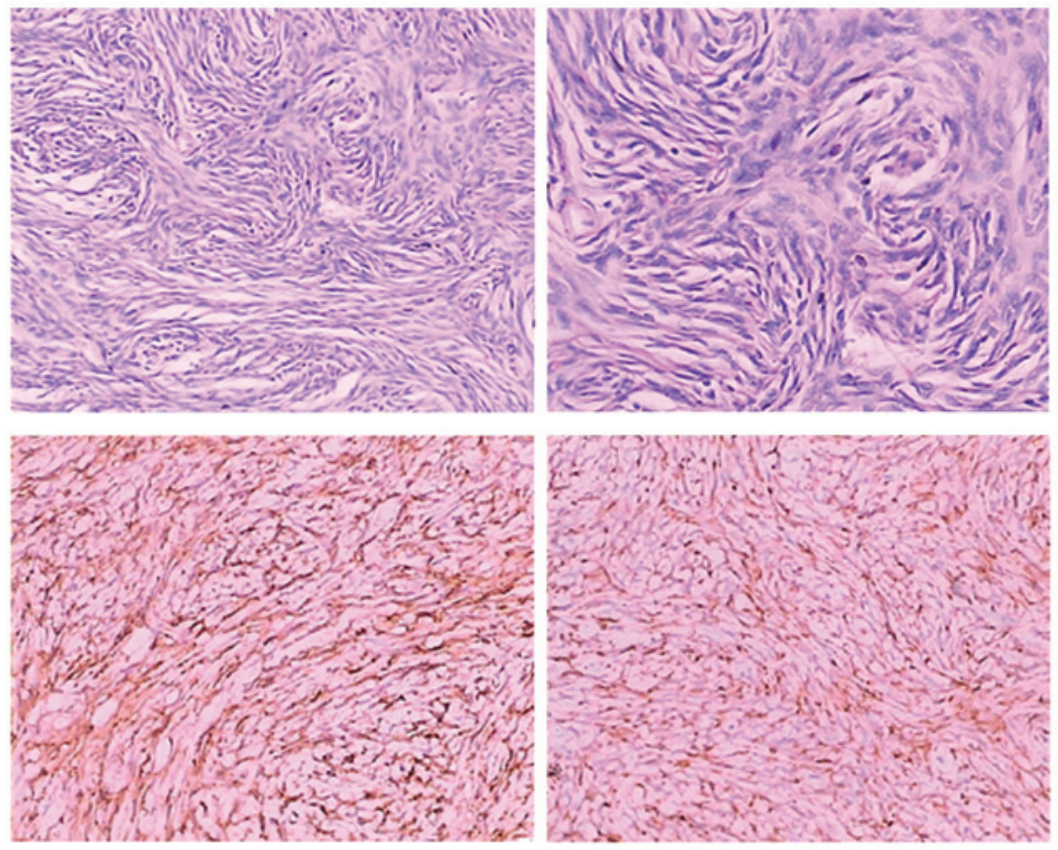

Figure 2. Hematoxylin-eosin staining of neoplastic cells of the prostate tumor. Magnification, x100 (upper left) and x200 (upper right). Expression of CD34 (lower left) and bcl-2 (lower right) in neoplastic cells, identified by immunohistochemistry (magnification, x100).

prostate SFT. Written informed consent was obtained from the patient.

\section{Case report}

A 46-year-old male with irritative lower urinary tract symptoms and increasing dysuria was found to have an enlarged, smooth and tenacious prostate on digital rectal examination. Serum prostate-specific antigen levels were within the normal limits (0.68 ng/ml; normal range, $0-4 \mathrm{ng} / \mathrm{ml})(17)$; however, the patient's maximum urinary flow rate was reduced to $10 \mathrm{ml} / \mathrm{s}$. The prostate was measured at $64 \times 56 \times 57 \mathrm{~mm}$, combined with multiple cystoliths (the largest of which was $\sim 6 \times 6 \mathrm{~mm}$ ), using images from a transabdominal ultrasound. In addition, the International Prostate Symptom Score was 13 (overall score range, 0-35), indicating a moderate grade of benign prostatic hyperplasia (moderate score range, 8-19) (18). The present case was initially diagnosed as benign prostatic hyperplasia, complicated with bladder calculus. The patient received cystoscopy and lithocystotomy per urethra, which was combined with doxazosin (oral dose, $4 \mathrm{mg} /$ day) and finasteride (oral dose, $5 \mathrm{mg} /$ day) treatment following surgery. However, the symptoms were not improved. Subsequently, a large circumscribed tumor within the prostate, which appeared to be focally intimate with the bladder neck and partially invading the urinary bladder was identified on a magnetic resonance imaging (MRI) scan (Fig. 1). Approximately two weeks later, TUR of the prostate (TURP) was performed and $\sim 80 \mathrm{~g}$ tissue was removed. Histopathological and immunohistochemical analyses were performed and it was identified that the neoplastic cells were spindle cells arranged in storiform. In addition, CD34 and bcl-2 were highly expressed in 
the tumor cells (Fig. 2). However, the cells were negative for CD117 (c-kit), S-100, discovered on GIST-1 (Dog-1), Ki-67 and CD68. Approximately 20 days subsequent to TURP, the patient exhibited a recurrence of aggravating dysuria, and a locoregional recurrence of tumor was identified by ultrasound and MRI. Subsequently, a nerve-sparing retropubic radical prostatectomy was performed, and the mass appeared to be well ablated, with no invasion of the bladder neck or pelvic wall identified. Postoperatively, the patient exhibited normal erectile and voiding function, with no locoregional recurrence identified in follow-ups over the subsequent 18 months. The final diagnosis of this lesion was borderline prostatic SFT.

\section{Discussion}

As a result of the lack of typical clinical presentations, ultrasound, MRI or computerised tomography (CT) are always required for the diagnosis of SFT lesions. However, SFTs cannot be definitively differentiated from other tumors by imaging alone. Specimens of prostatic SFT are frequently isolated from fine needle aspiration biopsies, TUR or open surgery. The tumors are characterized histologically by uniform spindle-shaped cells, which are arranged in storiform, herringbone or with a 'patternless' growth pattern of alternating hyper- and hypocellular areas, or a combination of these patterns (15). Furthermore, tumor cells are invariably positive for CD34, CD99 and bcl-2, but negative for S-100 protein, actin, desmin and epithelial markers, which therefore represent valuable diagnostic supports $(2,15)$. As previously reported, the diagnosis of SFTs is conclusively based on the histopathological and immunohistochemical characteristics of the tumor $(7,8,19)$. The present case did not significantly differ from those previously reported. The neoplastic cells were identified to be spindle cells, which were arranged in storiform. In addition, CD34 and bcl-2 were highly expressed, but the tumor was negative for CD117 (c-kit), S-100, Dog-1, Ki-67 and CD68. Therefore, the case was ultimately diagnosed as SFT.

Therapeutic strategies for the treatment of prostatic SFTs, include TUR, enucleation and complete tumor resection (cystoprostatectomy), radical prostatectomy, pelvic exenteration and pelvic tumor resection. Due to the fact that it is difficult to predict the clinical behaviour of SFTs, undergoing complete tumor resection currently has the greatest influence on prognosis, emphasizing the importance of resection margins (12).

In the present case, in view of the large size and rapid growth of the tumor following TURP, retropubic prostatectomy was performed, which was concerned with the preservation of sexual function. Following surgery, the patient exhibited normal erectile and voiding function, with no locoregional recurrence within the 18 month follow-up period.

\section{References}

1. Klemperer P and Rabin CB: Primary neoplasms of the pleura. A report of the five cases. Arch Pathol 11: 385-412, 1931.

2. Galosi AB, Mazzucchelli R, Scarpelli M, et al: Solitary fibrous tumour of the prostate identified on needle biopsy. Eur Urol 56: 564-567, 2009.

3. Parada Domínguez D, Peña González K, Morente Laguna V and Riu Ferrando F: Solitary fibrous tumor of the prostate. Actas Urol Esp 34: 119-121, 2010 (In Spanish).

4. Herawi M and Epstein JI: Solitary fibrous tumor on needle biopsy and transurethral resection of the prostate: A clinicopathologic study of 13 cases. Am J Surg Pathol 31: 870-876, 2007.

5. Chen KT: Hemangiopericytoma of the prostate. J Surg Oncol 35: 42-43, 1987.

6. Grasso M, Blanco S, Franzoso F, Lania C, Di Bella C and Crippa S: Solitary fibrous tumor of the prostate. J Urology 168: 1100, 2002.

7. Kelly PM and Baxter GM: Solitary fibrous tumour of the prostate. Brit J Radiol 71: 1086-1088, 1998.

8. Mentzel T, Bainbridge TC and Katenkamp D: Solitary fibrous tumour: Clinicopathological, immunohistochemical, and ultrastructural analysis of 12 cases arising in soft tissues, nasal cavity and nasopharynx, urinary bladder and prostate. Virchows Arch 430: 445-453, 1997.

9. Noguchi M, Hirabayashi Y, Kato S and Noda S: Solitary fibrous tumor arising from the prostatic capsule. J Urol 168: 1490-1491, 2002.

10. Pins MR, Campbell SC, Laskin WB, Steinbronn K and Dalton DP: Solitary fibrous tumor of the prostate: A report of 2 cases and review of the literature. Arch Pathol Lab Med 125: 274-277, 2001.

11. Reyes JW, Shinozuka H, Garry P and Putong PB: A light and electron microscopic study of a hemangiopericytoma of the prostate with local extension. Cancer 40: 1122-1126, 1977.

12. Sekine H, Ohya K, Kojima S and Mizuguchi K: Solitary fibrous tumor of the prostate. Int J Urol 8: 137-138, 2001.

13. Takeshima Y, Yoneda K, Sands N and Inai K: Solitary fibrous tumor of the prostate. Pathol Int 47: 713-717, 1997.

14. Westra WH, Gerald WL and Rosai J: Solitary fibrous tumor. Consistent CD34 immunoreactivity and occurrence in the orbit. Am J Surg Pathol 18: 992-998, 1994.

15. Nair B, Nambiar A, Hattangadi SB, Sukumar S and Saifuddin MS: Solitary fibrous tumour of prostate: Evaluation and management of a rare tumour. Scand J Urol Nephrol 41: 442-444, 2007.

16. Talvitie H, Aström K, Larsson O, Ahlén J, Bergh A and Egevad L: Solitary fibrous tumor of the prostate: A report of two cases. Pathol Int 61: 536-538, 2011.

17. Park KK, Lee SH, Choi YD and Chung BH: Optimal baseline prostate-specific antigen level to distinguish risk of prostate cancer in healthy men between 40 and 69 years of age. J Korean Med Sci 27: 40-45, 2012.

18. Roehrborn CG: Focus on lower urinary tract symptoms: Nomenclature, diagnosis, and treatment options: Highlights from the 5th international consultation on benign prostatic hyperplasia June 25-27, 2000, Paris, France. Rev Urol 3: 139-145, 2001.

19. Vodovnik A, Rogawski K and Bolton JF: A case of malignant solitary fibrous tumor of the prostate. Pathol Int 55: 807-808, 2005. 